\title{
Quantitative Examination of Reverse-Biased Semiconductor Devices using Off- Axis Electron Holography
}

\author{
A.C. Twitchett", R.E. Dunin-Borkowski* and P.A. Midgley* \\ * Department of Materials Science and Metallurgy, University of Cambridge, Pembroke Street, \\ Cambridge, CB2 3QZ, UK
}

The characterisation of device properties relies on quantitative determination of dopant distribution within the device structure. With the size of device features approaching the nanometre scale, the microelectronics industry requires characterisation techniques to image dopants with a spatial resolution of better than $10 \mathrm{~nm}$. Electron holography satisfies this need, generating two-dimensional maps of electrostatic potential with sub $10 \mathrm{~nm}$ resolution using a field emission gun transmission electron microscope (FEGTEM) [1]. The technique uses an electrostatic biprism (typically a quartz wire coated with gold) to overlap an electron wave that has passed through an electron-transparent sample with a wave that has passed through vacuum. The interference pattern (or hologram) that forms in the overlap region contains information about the phase change of the electron wave caused by the sample. The electrostatic potential in the sample can be determined through examination of the reconstructed holographic phase image [2]. A systematic series of experiments have been conducted using a Philips CM300 FEGTEM, illustrating the application of electron holography to a Si p-n junction. The electrostatic potential distribution was examined as a function of applied reverse bias and membrane thickness. A single-tilt biasing holder (Fig. 1a) was used to examine cleaved wedge specimens, which have spring contacts made to their front and back faces. A membrane of uniform thickness was micro-machined in the cleaved samples using focused ion beam (FIB) milling (Fig. 1b).

Figure 2a shows a $200 \mathrm{kV}$ holographic phase image obtained from an unbiased p-n junction prepared using a FIB workstation. The dark and bright contrast in the image corresponds to the $\mathrm{p}$ and n-type regions in the sample, respectively. An additional grey band along the edge of the sample is thought to result from the presence of an electrically dead layer that runs around its entire surface. A line trace showing the phase profile across the junction is plotted in Fig. 2b. Figure 2c shows the corresponding charge density profile determined from Fig. $2 \mathrm{~b}$ using Poisson's equation. Electrostatic fringing fields (Fig. 2d) were observed only outside reverse-biased cleaved wedge specimens before they were machined in the FIB workstation. Fringing fields were not observed outside either unbiased cleaved wedges or any FIB-prepared samples [3]. Membrane thicknesses were determined using convergent beam electron diffraction techniques and from holographic amplitude images. Discrepancies between results from these two techniques indicate the presence of a $30 \mathrm{~nm}$ amorphous layer at each membrane surface. Figure 3a shows a plot of the measured phase shift across a p-n junction as a function of applied reverse bias in a sample with a crystalline thickness of $390 \mathrm{~nm}$. These results are consistent with the sample containing an electrically active layer of thickness $340 \mathrm{~nm}$ with a built-in voltage of $0.9 \mathrm{~V}$. The remaining crystalline thickness of 50 $\mathrm{nm}$ is electrically dead. Through the examination of experimental results as a function of both bias and thickness, a semi-quantitative model for the cross-sectional structure of the sample can be deduced. Figure $3 \mathrm{~b}$ illustrates the layered structure suggested by the experimental results. 

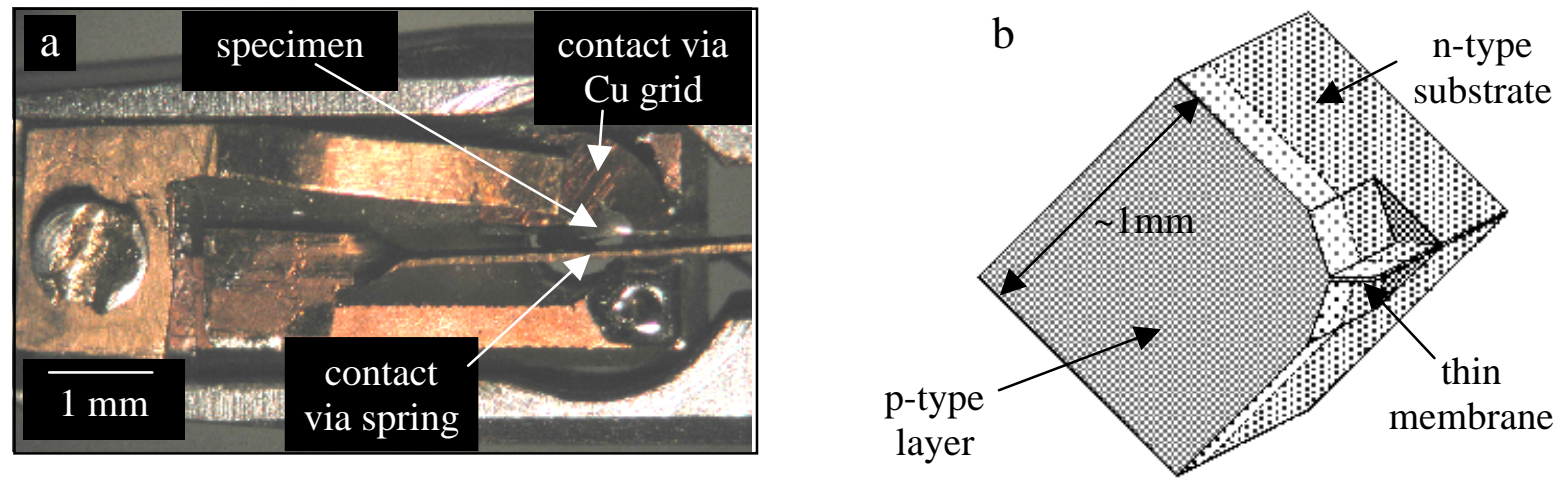

FIG. 1 (a) Electrical biasing holder; (b) Specimen geometry after cleaving and FIB milling.
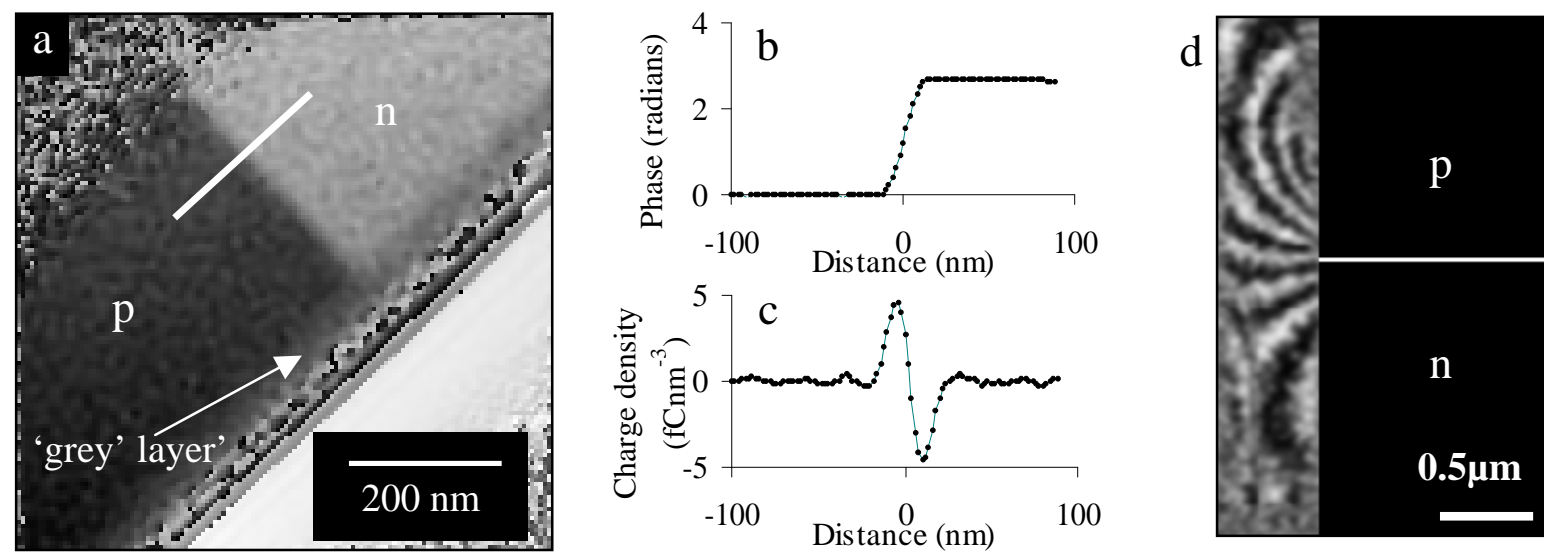

FIG. 2 (a) Reconstructed phase image of a Si p-n junction. (b) The phase and charge density across the junction. (c) Stray fields from non-FIB cleaved wedge sample at $2 \mathrm{~V}$ reverse bias.

a

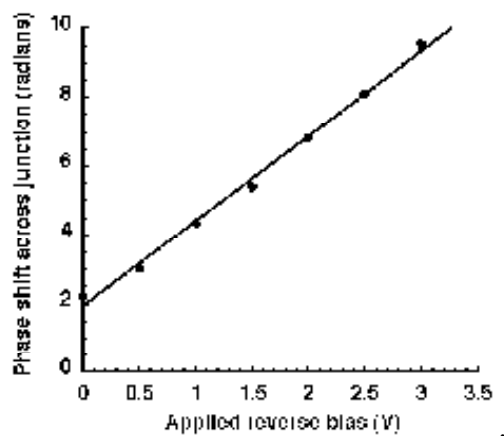

b

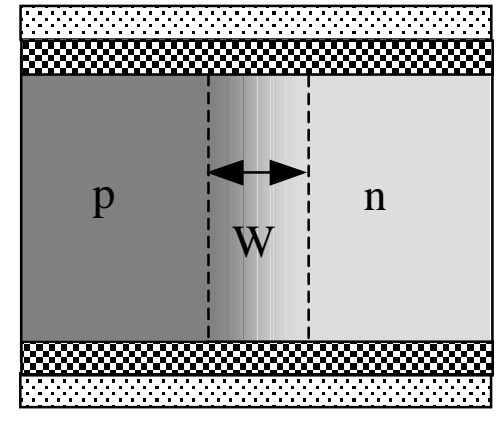

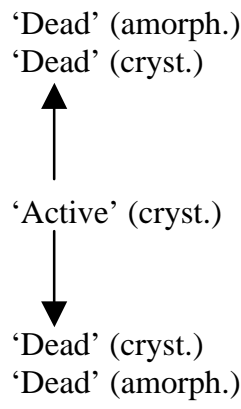

FIG. 3 (a) Phase change across junction as a function of reverse bias for sample with measured crystalline thickness of $390 \mathrm{~nm}$. (b) Model used to explain the results. W is the depletion width.

\section{References}

[1] P. A. Midgley, Micron 32 (2001) 167.

[2] W. D. Rau et al., Phys. Rev. Lett. 82 (1999) 2614.

[3] M. Beleggia et al., Proceedings of the 12th Conference on Microscopy of Semiconducting Materials (2001)

[4] We are grateful to the Royal Society, the EPSRC and the Worshipful Company of Armourers and Brasiers for financial support, and Philips Research (Eindhoven) for samples. 